in vivo $34: 1103-1110(2020)$

doi:10.21873/invivo.11881

\title{
Effect of Bone Powder/Mesenchymal Stem Cell/BMP2/Fibrin Glue on Osteogenesis in a Mastoid Obliteration Model
}

\author{
CHUL HO JANG ${ }^{1 *}$, GWANG WON $\mathrm{CHO}^{2,3^{*}}$ and AN-JI SONG ${ }^{2,3}$ \\ ${ }^{1}$ Department of Otolaryngology, Chonnam National University Medical School, Gwangju, Republic of Korea; \\ ${ }^{2}$ Department of Biology, College of Natural Science, Chosun University, Gwangju, Republic of Korea; \\ ${ }^{3}$ Department of Life Science, BK-21-Plus Research Team for Bioactive Control Technology, \\ Chosun University, Gwangju, Republic of Korea
}

\begin{abstract}
Background/Aim: This study aimed to prospectively compare the osteogenesis of bone powder $(B P)$ substances with and without mesenchymal stem cells (MSCs) and evaluate the synergistic effect of topically applied recombinant human bone morphogenic protein-2 (BMP2) on MSC-loaded BP using fibrin glue in a mastoid obliteration model. Materials and Methods: To determine the expression of osteocyte-specific genes, total RNA was isolated from three MSC groups: Untreated MSCs, MSCs cultured with BP, and MSCs cultured with $B P$ and $B M P 2$. Real-time polymerase chain reaction was carried out with specific primers of osteogenesis-related genes runt-related transcription factor 2, osteocalcin, osteoprotegerin, osterix, alkaline phosphatase, transforming growth factor beta, and type I collagen. Live/dead staining was also performed. To observe the adhesion of MSCs to the $B P, M S C s$ were treated with BP for 2 days and the surface was observed by scanning electron microscopy (SEM). Under general anesthesia, mastoid obliteration was performed in rats using three groups: treated with BP alone, BP/MSCs, and $B P / M S C / B M P 2$. Before decapitation at 8 weeks post operation, in vivo micro computed tomography (micro CT) was performed. The bullae were dissected, fixed, and decalcified. followed by dehydration, paraffin embedding, and staining by hematoxylin and eosin and Masson's trichrome. Results: SEM
\end{abstract}

This article is freely accessible online.

*These Authors contributed equally to this article.

Correspondence to: Chul Ho Jang, MD, Ph.D., Department of Otolaryngology, Chonnam National University Hospital, Gwangju, Republic of Korea, Tel: +82 622206774, e-mail: chulsavio@

Key Words: Bone powder, mesenchymal stem cell, bone morphogenic protein, osteogenesis. showed the MSCs to be well-attached to the superficial area of the BP. The expression of osteocyte-specific genes was the highest in the MSCs cultured with BP and BMP2, followed by cultured with BP only, and untreated MSCs. The $B P / M S C / B M P 2$ group showed the highest radiodensity of bullae in microCT analysis. The microCT findings revealed that the BP/MSC/BMP2 group showed the most enhanced osteogenesis of the scaffold compared to the other two groups. No significant difference was found in osteoconductive osteogenesis between the control and BP/MSC groups. However, the $B P / M S C / B M P 2$ group showed significantly enhanced osteoconductive osteogenesis and osteoinductive change of the BP as shown by hematoxylin and eosin staining. Histomorphometry of osteogenesis revealed that the difference between the BP/MSC/BMP2 group and the other two groups was statistically significant. Conclusion: A small amount of BMP2 is necessary during MSC loading to enhance the osteogenesis of BP and avoid complications associated with high doses of BMP2. These results may be applicable to mastoid obliteration in clinical practice.

The primary goal of surgical treatment for cholesteatoma or chronic active otitis media is the complete removal of the cholesteatoma and prevention of recurrence. Following canal wall-down tympanomastoidectomy, obliteration of the mastoid and epitympanic spaces can be performed using autologous material such as autologous tissues (1-6), homograft materials $(4,7,8)$, or biocompatible materials (811). Among autogenous tissues, bone powder (pate) can easily be used if the surgeon can collect bone pate during mastoidectomy $(1,12,13)$. However, it is difficult to collect sufficient bone powder during revision surgery or extended cholesteatoma. In this situation, demineralized bone powder (BP) can also be used instead of autogenous bone powder. Demineralized BP is different from demineralized bone matrix with regard to size and porosity. It has been used as 
in vivo $34: 1103-1110(2020)$

Table I. Oligonucleotides used for quantitative polymerase chain reaction.

\begin{tabular}{|c|c|c|c|c|}
\hline Gene & Encoded protein & Forward primer $\left(5^{\prime} \rightarrow 3^{\prime}\right)$ & Reverse primer $\left(5^{\prime} \rightarrow 3^{\prime}\right)$ number & Gene accession \\
\hline RUNX2 & $\begin{array}{l}\text { Runt-related transcription } \\
\text { factor } 2 \text { isoform B }\end{array}$ & TCTGACCGCCTCAGTGATTT & TGCCTGGGGTCTGTAATCTG & NM_001024630 \\
\hline OCN & Osteocalcin preproprotein & CACCGAGACACCATGAGAGC & CTGCTTGGACACAAAGGCTGC & NM_199173.6 \\
\hline OSX & Transcription factor SP7 isoform & AGGAGGAAGTTCACTATGGCTC & TAAAGGGGGCTGGATAAGCATC & NM_001173467.3 \\
\hline ALP & $\begin{array}{l}\text { Alkaline phosphatase, } \\
\text { tissue-nonspecific isozyme } \\
\text { isoform } 1 \text { preproprotein }\end{array}$ & GCACCATGAAGGAAAAGCCA & TGTGAAGACGTGGGAATGGT & NM_000478 \\
\hline TGFB & $\begin{array}{l}\text { Transforming growth factor } \\
\text { beta-1 proprotein preproprotein }\end{array}$ & CAAGTGGACATCAACGGGTTC & TCCGTGGAGCTGAAGCAATAG & NM_000660.7 \\
\hline COL1A1 & $\begin{array}{l}\text { Collagen alpha- } 1(\mathrm{I}) \text { chain } \\
\text { preproprotein }\end{array}$ & CTGGAAGAGTGGAGAGTACTGG & GGAATCCATCGGTCATGCTCT & NM_000088.3 \\
\hline ACTB & $\beta$-Actin, cytoplasmic 1 & ATCCGCAAAGACCTGTACGC & TCTTCATTGTGCTGGGTGCC & NM_001101 \\
\hline
\end{tabular}

an osteoinductive biomaterial for enhancing osteogenesis in long bones and dental implants for maxillary sinus floor augmentation $(14,15)$. The BP collected by the sheet collector during mastoidectomy contains minerals. However, mastoid obliteration has the following limitations. The osteogenesis of obliterated BP is very slow. In our experience, even after 1 year, the temporal bone will have bone density much lower than that of the surrounding normal bone. Therefore, osteoinduction is necessary to promote BP osteogenesis. One of the methods for enhancing osteoinduction of BP is loading mesenchymal stem cells (MSCs) into the BP.

MSCs can be differentiated into several cell types, such as osteoblasts, myoblasts, neural cells, and chondrocytes (16). To date, the efficacy of BP with MSCs in mastoid obliteration is poorly understood. With regard to anatomical structure, the mastoid cavity is different from that of calvarial bone or long bone defects in which neighboring bone marrow is present close to the bone defect. Therefore, after mastoid obliteration, the healing time for osteogenesis takes longer than that expected. We hypothesized that using BP with MSCs might improve osteogenesis following mastoid obliteration.

Fibrin glue, fibrinogen, and thrombin are isolated from blood and enable the fabrication of completely autologous and inexpensive scaffolds. They are bioactive glues commonly used during surgery and serve as natural scaffolds, suitable for MSCs and biomolecule delivery systems $(17,18)$. Bone morphogenic protein 2 (BMP2) a member of the transforming growth factor family. Recombinant human BMP2 is approved by the US Food and Drug Administration and is clinically available. However, clinical application of high doses of BMP2 resulted in unexpected side-effects (19) Therefore, if a small amount of BMP2 has a synergistic effect on the osteogenesis of MSCseeded BP, it can be considered an ideal combination.
This study aimed to prospectively compare the osteogenesis of BP substances with and without human MSCs and evaluate the synergistic effect of topically applied recombinant human BMP2 on MSC-loaded BP using fibrin glue in a rat mastoid obliteration model.

\section{Materials and Methods}

Scaffold preparation and MSC treatment. Demineralized BP (Cellumed Co., Seoul, Republic of Korea) was used. Fibrin glue was obtained from a Greenplast kit (Green Cross Corp., Seoul, Republic of Korea). To fabricate MSC-loaded BP, human bone marrow-derived MSCs (CEFO, Cell Engineering for Origin, Korea) were cultured in growth medium containing low glucose Dulbecco's modified Eagle's medium (Gibco, USA) supplemented with $1 \%$ penicillin, $1 \%$ streptomycin, and $10 \%$ fetal bovine serum (FBS; Gibco). MSCs ( $5^{\prime}$ $10^{4}$ cells/24-well plate) were then seeded onto culture dishes containing BP (1.5 mg) and cultured for 2 days. After $24 \mathrm{~h}$ of seeding, the cells were treated with $100 \mathrm{ng} / \mathrm{ml}$ recombinant human BMP2 (CowellMedi Co., Pusan, Republic of Korea) and incubated for an additional $24 \mathrm{~h}$ in a humidified $\mathrm{CO}_{2}$ incubator maintained at $37^{\circ} \mathrm{C}$.

In vitro study. In order to determine the expression of osteocytespecific genes runt-related transcription factor 2 (RUNX2), osteocalcin $(O C N)$, osteoprotegerin $(O P G)$, osterix $(O S X)$, alkaline phosphatase $(A L P)$, transforming growth factor beta $(T G F B)$, type I collagen $(C O L 1 A 1)$ in osteogenesis using BP, total RNA was isolated from three MSC groups: Untreated MSCs, MSCs cultured with BP, and MSCs cultured with BP and BMP2, using RNeasy Mini Kit (Qiagen Co., Hilden, Germany). Synthesis of cDNA samples was carried out using $200 \mathrm{ng}$ of each total RNA (PrimeScript II $1^{\text {st }}$ Strand cDNA Synthesis Kit; TAKARA, Tokyo, Japan). The quantitative polymerase chain reactions were performed with specific gene primers using Power SYBR Green PCR Master Mix (Applied Biosystems, Carlsbad, CA, USA). Each value was normalized to the expression of $\beta$-actin. All the primers were produced by GenoTech (GenoTech Corp., Daejeon, Republic of Korea) and are summarized in Table I.

For live and dead staining, MSCs cultured with bone powder were washed with phosphate-buffered saline (PBS). Then MSCs 
A

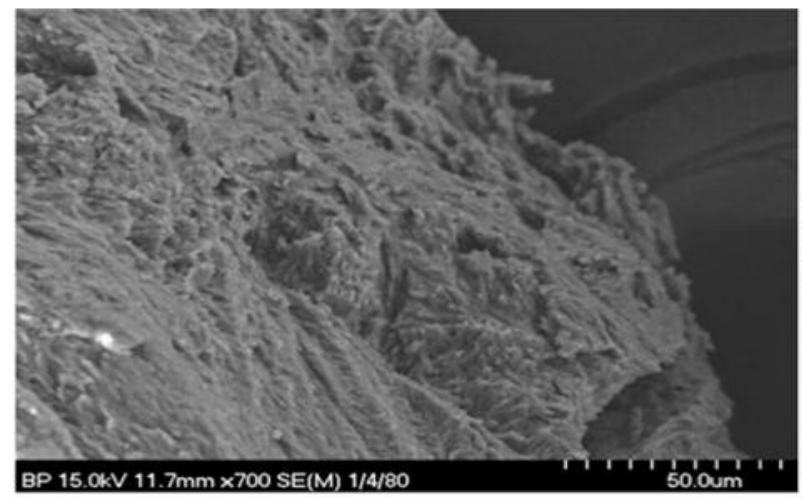

B Plated MSCs
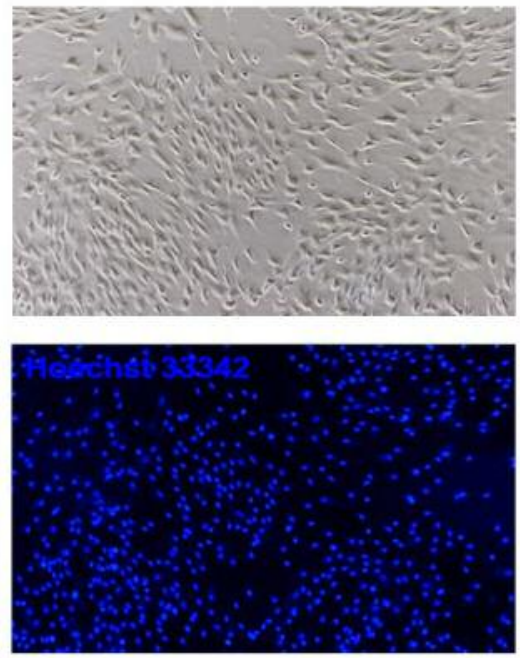

$\mathrm{BP}$
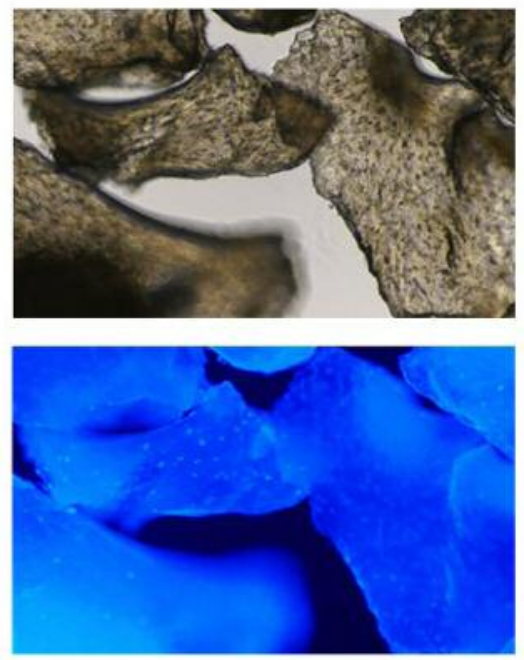

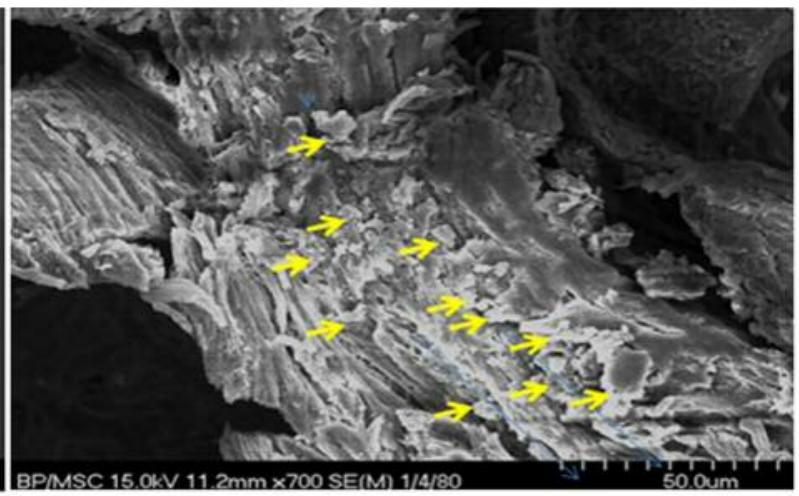

BP/BMP2
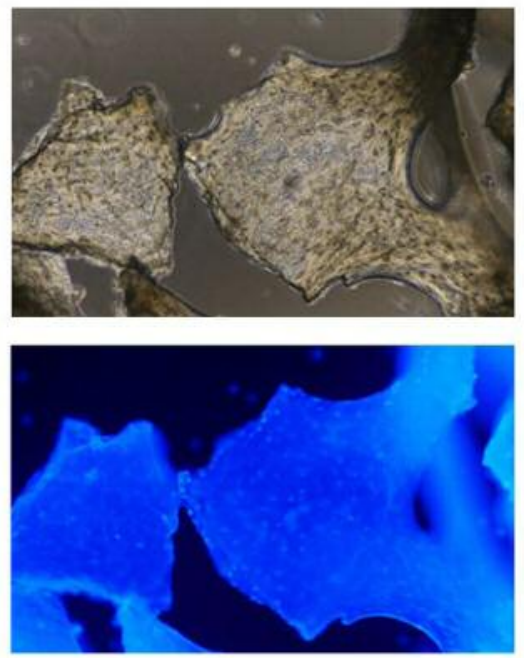

Figure 1. Surface detail revealed by scanning electron microscopy (A) and fluorescent microscopy. A: On day 3, mesenchymal stem cell (MSC)-loaded bone powder (BP) scaffold shows MSCs on the surface and the very superficial parts of the BP (arrows). B: Live/dead staining. Both BP and BP/bone morphogenic protein 2 (BMP2) group showed live MSCs attached to the surface. Dead cells (red color by propidium iodide) were not found.

were stained with 1:1,000 diluted Hoechst 33342 (blue color for live cell staining; Molecular Probes Inc. MA, USA) and $1 \mu \mathrm{g} / \mathrm{ml}$ propidium iodide (red color for dead cell staining; Molecular Probes Inc.) in PBS for $15 \mathrm{~min}$. The stained cells were visualized under a fluorescence microscope (Nikon Eclipse 80Ti microscope, Nikon, Japan) for qualitative testing of live and dead cells.

Scanning electron microscopy (SEM) to confirm the adhesion of MSCs to $B P$. To observe the adhesion of MSCs to BP, MSCs were treated with and without BP and incubated for 2 days. The cells were then fixed overnight using $2.5 \%$ glutaraldehyde solution and subjected to serial dehydration using an alcohol solution. The BP and BP/MSC samples were dried and sputter-coated with platinum. The surface images were evaluated using an S-4700 field emission scanning electron microscope (FE-SEM; Hitachi, Tokyo, Japan). The SEM measurements were for performed in accordance with the manufacturer's instructions.

Surgical procedure for mastoid obliteration in a rat model. Before in vivo implantation of BP/MSC/BMP2 (group II), $40 \mathrm{ml}$ of BMP2
$(250 \mathrm{mg} / \mathrm{ml}$ ) was pipetted onto the MSCs on BP after removal of the culture medium, and left for $5 \mathrm{~min}$ to penetrate then the $\mathrm{BP} / \mathrm{MSC}$ layer was coated by fibrin glue, which was also applied to the other groups. In the BP/MSC/BMP2 group, it was topically applied with the aid of a collagen sponge with BMP2, easily adhered to the MSC-loaded BP scaffold.

Animal experiments were approved by the Institutional Animal Care and Use Committee of the University Medical School (Permit Number: IACUC-H-2018-57). A total of 15 healthy SpragueDawley rats weighing 200-250 g (Samtakobio, Suwon, Kyungki, Republic of Korea), with normal tympanic membranes and Preyer's reflex were used in this study. The tympanic membrane of rats was examined using otomicroscopy. To prepare rat bullae for receiving the BP (control group), BP/MSC (group I), and BP/MSC/BMP2 (group II), periauricular hairs were shaved and antiseptic painting was performed using povidone solution under general anesthesia was induced by isoflurane inhalation. Prior to incision, dental lidocaine $(1: 100,000)$ was administered around the auricle. A skin incision was made around the auricle to expose the right bulla. The 


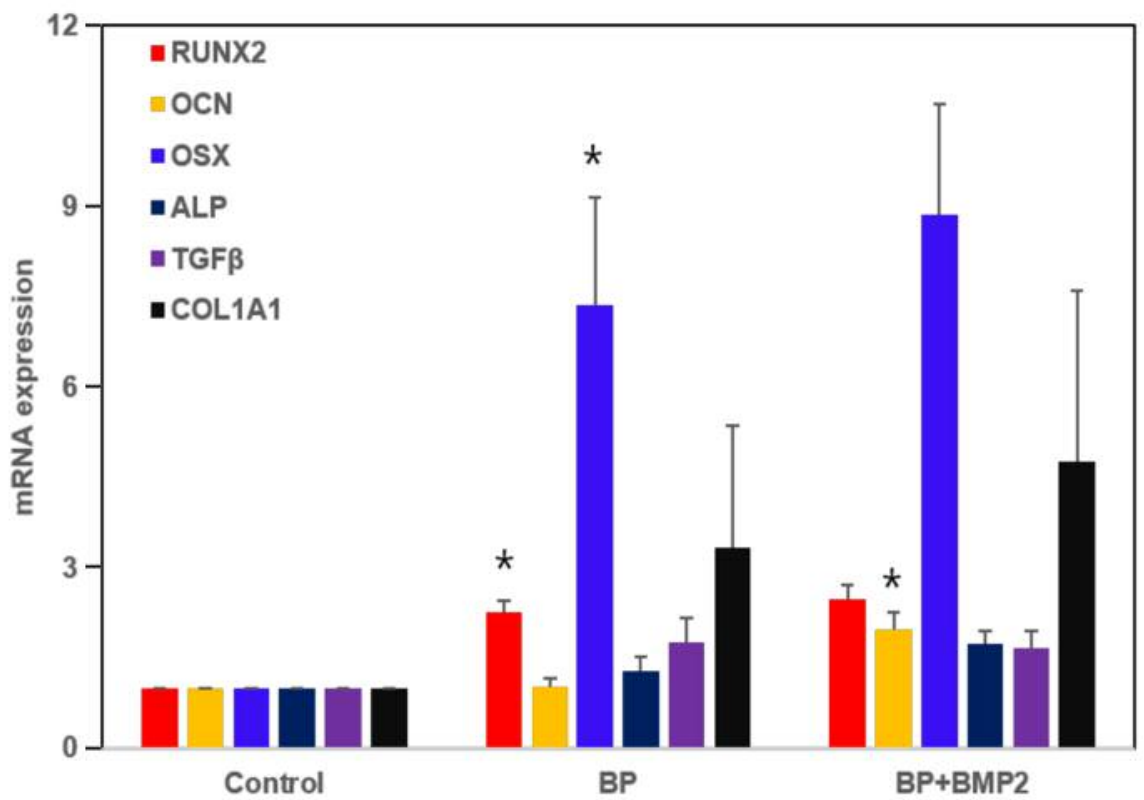

Figure 2. The expression of osteocyte-specific genes in bone powder substances with mesenchymal stem cells (MSCs) was used to evaluate the synergistic effect of topically applied recombinant human bone morphogenic protein-2 (BMP2). Control: MSCs alone. BP: Bone powder with MSCs. BP+BMP2: Bone powder with BMP2 and MSCs. The gene expressions of the BP group were compared with those of the control and those of the BM+BMP2 group with the BP group. RUNX2: Runt-related transcription factor 2 isoform B; OCN: osteocalcin preproprotein; OSX: transcription factor SP7 isoform; ALP: alkaline phosphatase, tissue-nonspecific isozyme isoform 1 preproprotein; TGFB: transforming growth factor beta-1 proprotein preproprotein; COL1A1: collagen alpha-1(I) chain preproprotein. Data are the mean $\pm S D(n=4)$. *Significantly different at $p<0.05$ by $t$-test.

subcutaneous tissue was dissected by a fine dental periosteal elevator and the blood was coagulated by portable cordless bovie (Pocket Cautery, Pioneer Co., Canterbury, UK). The lateral wall of the bulla cavity was opened by drilling and flushed with PBS to remove bone dust in the bulla cavity as residual bone dust can interfere with bone formation as an artifact. The bulla was filled with $\mathrm{BP} /$ fibrin glue (control group, $\mathrm{n}=5$ ), BP/MSC/fibrin glue $(\mathrm{n}=5)$, or BP/MSC/BMP2/fibrin glue ( $\mathrm{n}=5)$. The skin incision was sutured by autoclip (Jeung Do Bio \& Plant co, Seoul, Republic of Korea).

In vivo microCT evaluation. To assess new bone formation, rats in each group were sacrificed by decapitation 8 weeks after bulla obliteration. Before decapitation, in vivo microCT evaluation was carried out using a Quantum GX $\mu \mathrm{CT}$ imaging system (PerkinElmer, Hopkinton, MA, USA) at Korea Basic Science Institute (KBSI, Gwangju, Republic of Korea). The X-ray source was set at $90 \mathrm{kV} / 80$ $\mu \mathrm{A}$ with a field of view of $45 \mathrm{~mm}$ (voxel size: $90 \mu \mathrm{m}$, scan mode: high resolution, scan time: $4 \mathrm{~min}$ ) and an approximate dose (X-ray) of $162 \mathrm{mGy}$ radiation per scan. To quantify and objectively compare the amount of mineralization by osteogenesis, we cropped the obliterated bulla from the axial microCT images and visualized the central portion of each scaffold. From the axial microCT image, we measured the mean grey values (Hounsfield units) using ImageJ 1.52a (National Institute of Health, Bethesda, MD, USA).

Histopathological evaluation. The decapitation was performed 8 weeks after the surgery. The bullae were harvested and fixed in $10 \%$ formalin solution. All bullae were decalcified using $10 \%$ EDTA solution. After decalcification, each bulla was dissected using a surgical knife and the gross appearance of the implanted scaffold was observed using a stereomicroscope. After observing the gross appearance, serial dehydration was performed using alcohol, followed by paraffin embedding. The sections were stained using hematoxylin-eosin (HE) and Masson's trichrome (MT) stain. The density of osteogenesis obtained by MT staining was quantified using ImageJ program and compared between groups.

Statistical analysis. The statistical analysis was initially performed using ANOVA and significant differences were resolved by the Tukey's test. Differences were considered statistically significant when $p$-values were less than 0.05 .

\section{Results}

Surface characteristics by SEM and live/dead staining. The SEM result showed the MSCs to be well-attached to the superficial area of the BP (Figure 1, indicated by arrows). According to live and dead staining, cells in all the groups were well-attached to the BP and mostly alive in the BP/MSC and BP/MSC/BMP2 groups (Figure 1B). No dead cells were detected by propidium iodide staining (data not shown). Expression of osteocyte-specific genes. Expression of OSX, COL1A1 and RUNX2 genes was increased in both BP/MSC and BP/BMP2 groups compared with the control group (MSCs alone). OSX gene expression was highest compared with the control group. Expression of $O S X, C O L 1 A 1$, and 

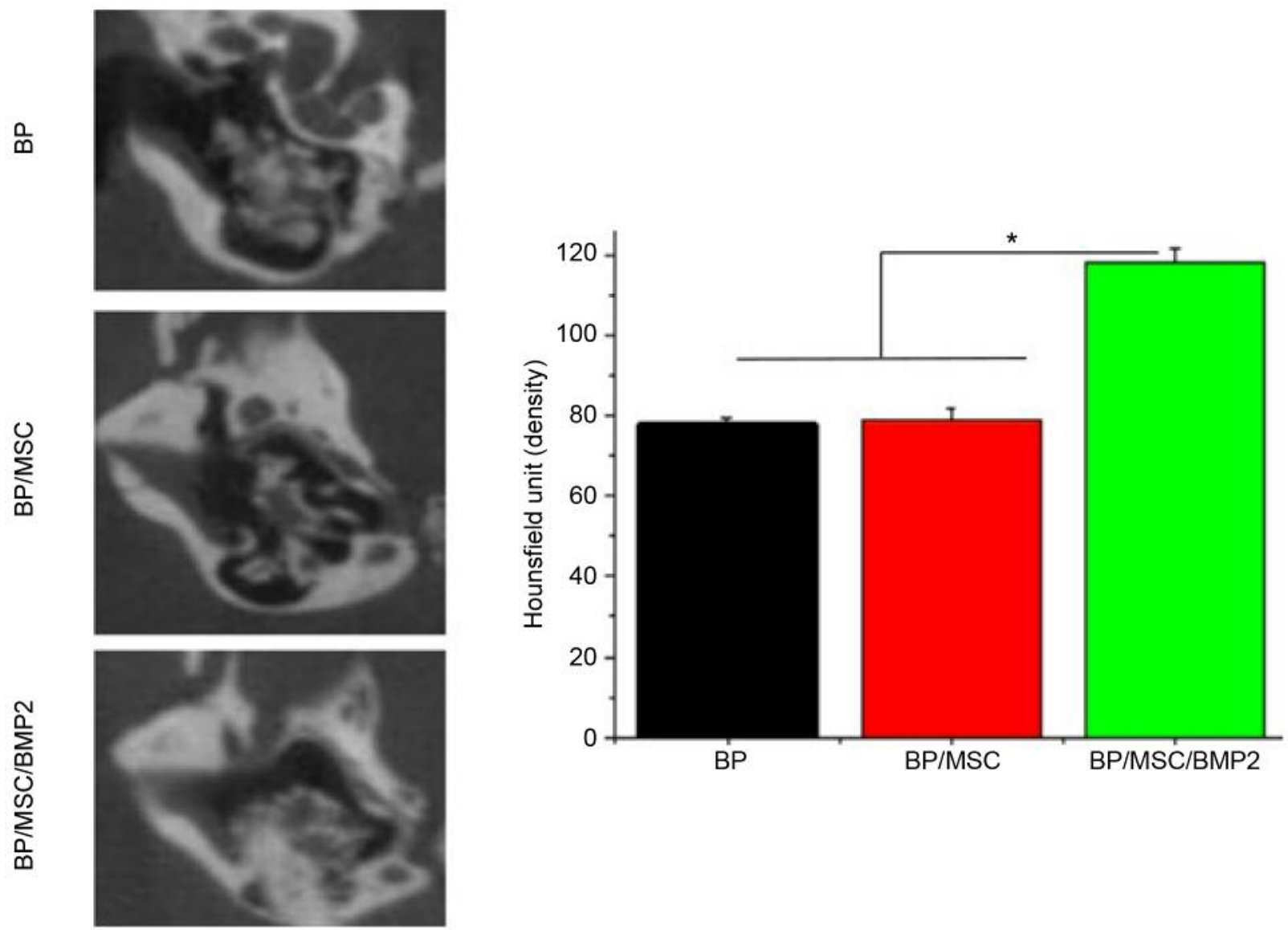

Figure 3. Micro-computed tomographic radiodensity of bone measured by the Hounsfield scale revealed that the group in which bullae were obliterated with bone powder with mesenchymal stem cells and recombinant human morphogenic protein-2 (BP/MSC/BMP2) had significantly higher radiodensity than the BP or BP/MSC groups. No significant difference was observed between the BP and BP/MSC groups. *Significantly different at $p<0.05$.

OCN genes was found to increase more in the BP/BMP2 group than the BP/MSC group (Figure 2).

Post-operative complications. All rats recovered from general anesthesia after the operation. None of the rats showed any wound infection or post-operative complications.

Evaluation of microCT results. Micro-CT imaging revealed that the BP was uniformly distributed in the bulla cavity in all the groups. With regard to radiodensity/radiopacity, which reflects mineralization, microCT revealed insignificant differences between the BP and BP/MSC groups. However, the radiodensity was significantly higher in the $\mathrm{BP} / \mathrm{MSC} / \mathrm{BMP} 2$ group than in the control and $\mathrm{BP} / \mathrm{MSC}$ groups. Osteogenesis was evident in the interface area between the host bone and scaffold. The Hounsfield scale, measured using ImageJ, showed the differences between the BP/MSC/BMP2 group and the control and $\mathrm{BP} / \mathrm{MSC}$ groups were significant (Figure 3).
Histological results. It was difficult to find any differences in osteoconductive osteogenesis between the control and $\mathrm{BP} / \mathrm{MSC}$ groups. However, the BP/MSC/BMP2 group showed significantly enhanced osteoconductive osteogenesis and osteoinductive change of the BP as shown by HE staining. Histomorphometry revealed a statistically significant difference between the BP/MSC/BMP2 group and the other two groups $(p<0.05)$ (Figure 4A). Enhanced osteoconductive osteogenesis in the BP/MSC/BMP2 group was also supported by MT staining. The increased intensity of blue in MT staining as measured by Image $\mathrm{J}$ indicated enhanced osteogenesis (Figure 4B). The intensity of the blue staining was statistically higher in the $\mathrm{BP} / \mathrm{MSC} / \mathrm{BMP} 2$ group than in the control and $\mathrm{BP} / \mathrm{MSC}$ groups $(p<0.05)$.

\section{Discussion}

To date, three BPs are commercially available for surgery: Homograft cortical BP, cancellous BP, and demineralized BP. 
A

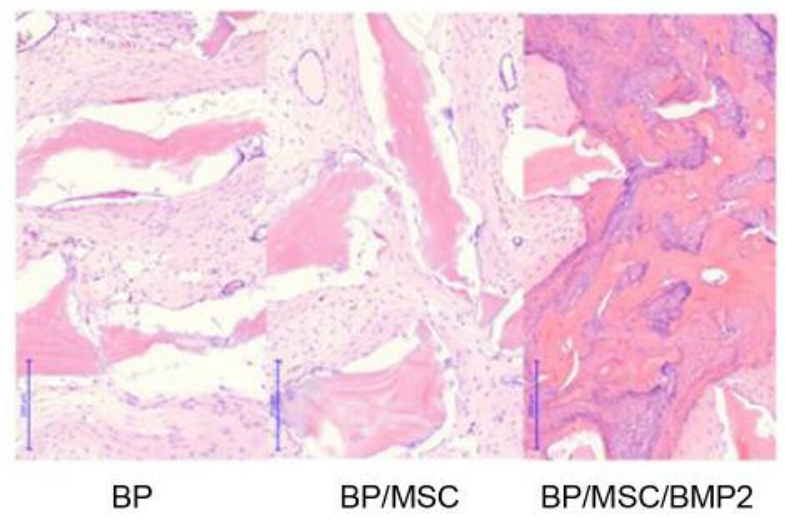

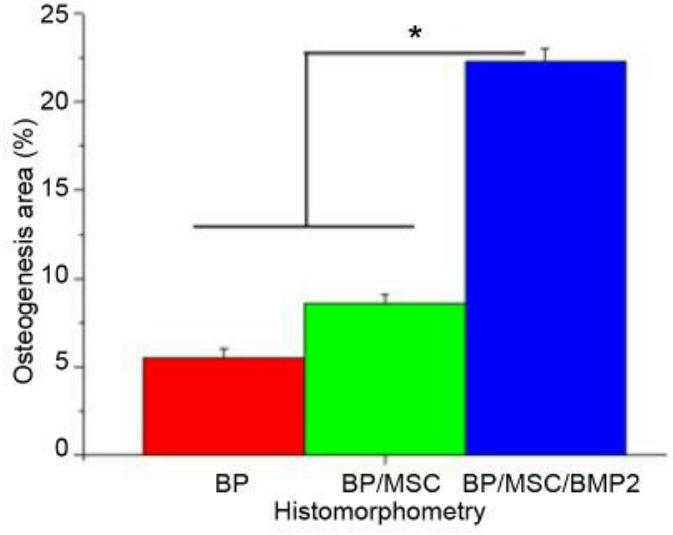

B
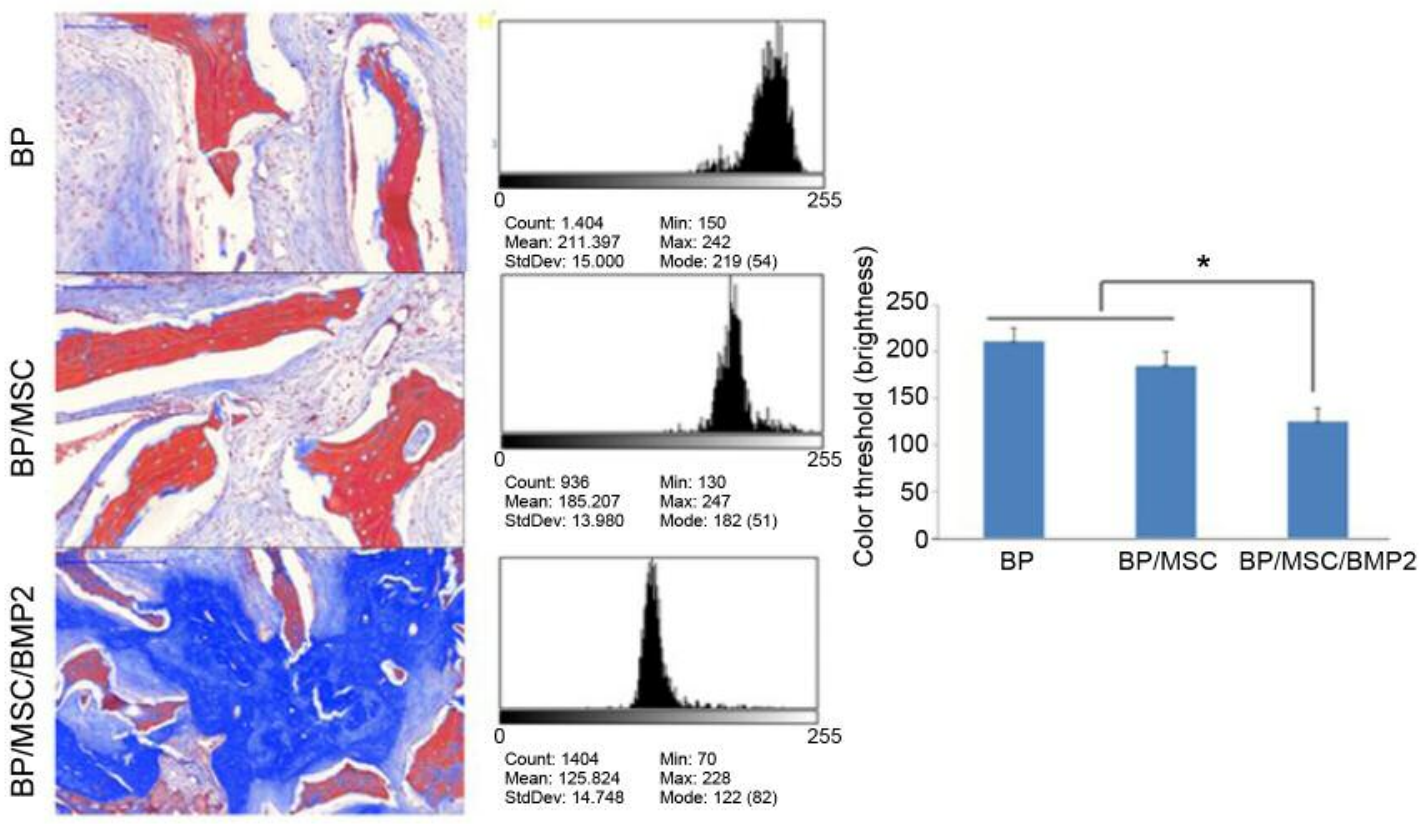

Figure 4. Changes in osteogenesis in bullae obliterated with bone powder (BP) alone and with mesenchymal stem cells (MSC) and recombinant human bone morphogenic protein-2 (BMP2). A: Histological results from hematoxylin and eosin staining showed BM/MSC/BMP2 significantly enhanced formation of new bone and osteoinductive change of the BP to mature bone compared to both the BP and BP/MSC groups. Histomorphological findings showed a mild, non-significant increase in the BP/MSC group compared with the BP group. In contrast, the $B P / M S C / B M P 2$ group showed a significant increase compared to the other two groups. B: Masson's trichrome staining results were similar to those of HE staining; the blue color indicates newly formed bone collagen tissue and its intensity was measured using ImageJ. The color threshold increases with the intensity/brightness of the blue color. Its intensity was significantly higher in the BP/MSC/BMP2 group than in the other two groups. Original magnification, $\times 100 . *$ Significantly different at $p<0.05$.

It is well known that the majority of antigenic substances present in demineralized BP are removed during the demineralization process. Therefore, demineralized BP does not evoke the host immune response (4). Consequently, we did not observe any inflammatory reaction throughout the histopathological examination. In this study, we compared osteogenesis among three groups. The quantitative polymerase chain reaction analysis of osteocyte-related genes showed increased expression in $\mathrm{BP} / \mathrm{MSC}$ and $\mathrm{BP} / \mathrm{MSC} / \mathrm{BMP} 2$ groups. However, no significant difference was shown between
$\mathrm{BP} / \mathrm{MSC}$ and $\mathrm{BP} / \mathrm{MSC} / \mathrm{BMP} 2$, suggesting that MSCs grown on BP were stimulated into osteogenic differentiation, while BMP2 treatment did not significantly stimulate or disrupt differentiation in vitro.

Similarly, histological results showed slightly enhanced osteogenesis in the BP/MSC group. However, contrary to our expectations, we did not observe any significant acceleration of osteogenesis in the BP/MSC group in microCT. The $\mathrm{BP} / \mathrm{MSC} / \mathrm{BMP} 2$ group showed significant improvement in osteogenesis. Theoretically, osteoinductive materials are 
believed to exist in demineralized BP but they are estimated to be present in extremely limited quantities. In this in vitro study, the expression of bone differentiation-related genes in the BP/MSC/BMP2 group was found to be lower than that expected. This difference can be attributed to the amount of BMP2 used in the in vitro and in vivo experiments.

During MSC loading, BP, cell density, and hydrophilic properties, volume, and surface of the scaffold play important roles in the attachment and distribution of MSCs in the scaffold (20). Interconnectivity with sufficient porosity is very important for cell penetration into the scaffold (21). In the present study, we identified MSCs as being well-attached to the surface of the BP, exhibiting a good survival state as suggested by the SEM and live/dead staining results.

In the present study, we used fibrin glue in all the groups to prevent the detachment of MSCs from the BP. As a bioadhesive, fibrin glue also facilitates surgical handling during mastoid obliteration and adhesion to the walls of the bulla cavity in the rat. Moreover, it improved the stability of the MSCs attached to the surface of the BP during the experiment and enhanced topical drug application to the scaffolds (22). BMPs act on the differentiation of MSCs into osteoblasts and are important during osteogenesis. According to Marden et al., recombinant human BMP2 reconstituted with collagenous bone matrix induced more bone formation than did collagenous bone matrix alone or demineralized bone matrix (23). In addition, Groeneveld et al. reported that irrespective of implanted demineralized bone matrix or demineralized BP, new bone formation was most active in the area adjoining the host bone (24). Consistent with this result, we observed that the most active new bone formation in the $\mathrm{BP} / \mathrm{MSC} / \mathrm{BMP} 2$ group occurred at the interface of the host bone as shown by HE and MT staining. BP/BMP2 alone can also be used; however, in this case, a larger amount of BMP2 is required. High doses of BMP2 may lead to complications such as inflammation (25). Therefore, when used together with MSCs, the need to use only a small amount of BMP2 is advantageous.

\section{Conclusion}

There is a limitation with the use of MSCs alone in promoting osteogenesis of BP for mastoid obliteration. Therefore, we confirmed that a small amount of recombinant human BMP2 is necessary during MSC-loading to enhance osteogenesis of BP and avoid complications associated with the use of high doses of BMP2. These results may be applicable to clinical practice for mastoid obliteration.

\section{Conflict of Interest}

The Authors have no conflicts of interest to declare.

\section{Author's Contributions}

C.H.J. designed the research, G.W.C. and A.S. collected tissue samples and performed in vitro experiments. C.H.J. performed in vivo experiments and microCT examination. C.H.J. and G.W.C. analyzed data, and wrote the article.

\section{Acknowledgements}

This study was financially supported by Chonnam National University (Grant number: 2016-2887) and by a grant from the Basic Science Research Program through the National Research Foundation of Korea (NRF) funded by the Ministry of Education, Science and Technology (NRF-2018R1D1A1B07048074) and supported by the National Research Foundation of Korea (NRF) Grant funded by the Ministry of Science and ICT for Bioinspired Innovation Technology Development Project (NRF-2018M3C1B7021997).

\section{References}

1 Irving RM, Gray RF and Moffat DA: Bone pate obliteration or revision mastoidectomy: A five-symptom comparative study. Clin Otolaryngol Allied Sci 19(2): 158-160, 1994. PMID: 8026096. DOI: 10.1111/j.1365-2273.1994.tb01202.x

2 Shea MC Jr., Gardner G Jr. and Simpson ME: Mastoid obliteration with bone. Otolaryngol Clin North Am 5(1): 161172, 1972. DOI: 10.1089/ten.TEB.2015.0357

3 Geerse S, Ebbens FA, de Wolf MJ and van Spronsen E: Successful obliteration of troublesome and chronically draining cavities. J Laryngol Otol 131(2): 138-143, 2017. PMID: 28069077.

4 Bauer TW and Muschler GF: Bone graft materials. An overview of the basic science. Clin Orthop Relat Res 371: 10-27, 2000. PMID: 10693546.

5 Decher H: Reduction of radical cavities by homologous cartilage chips. Laryngol Rhinol Otol 64(8): 423-426, 1985. PMID: 4046693.

6 Montandon P, Benchaou M and Guyot JP: Modified canal wallup mastoidectomy with mastoid obliteration for severe chronic otitis media. ORL J Otorhinolaryngol Relat Spec 57(4): 198-201, 1995. PMID: 7478453. DOI: 10.1159/000276739

7 Leatherman BD and Dornhoffer JL: The use of demineralized bone matrix for mastoid cavity obliteration. Otol Neurotol 25(1): 22-25; discussion 25-26, 2004. PMID: 14724487.

8 Leatherman BD, Dornhoffer JL, Fan CY and Mukunyadzi P: Demineralized bone matrix as an alternative for mastoid obliteration and posterior canal wall reconstruction: Results in an animal model. Otol Neurotol 22(6): 731-736, 2001. PMID: 11698788. DOI: 10.1097/00129492-200111000-00004

9 Yung MM and Karia KR: Mastoid obliteration with hydroxyapatite-the value of high resolution CT scanning in detecting recurrent cholesteatoma. Clin Otolaryngol Allied Sci 22(6): 553-557, 1997. PMID: 11698788. DOI: 10.1097/ 00129492-200111000-00004

10 Zippel N, Schulze M and Tobiasch E: Biomaterials and mesenchymal stem cells for regenerative medicine. Recent Pat Biotechnol 4(1): 1-22, 2010. PMID: 20201799. DOI: 10.2174/ 187220810790069497

11 Vos J, de Vey Mestdagh P, Colnot D, Borggreven P, Orelio C and Quak J: Bioactive glass obliteration of the mastoid significantly 
improves surgical outcome in non-cholesteatomatous chronic otitis media patients. Eur Arch Otorhinolaryngol 274(12): 4121-4126, 2017. PMID: 28956143. DOI: 10.1007/s00405-017-4757-7

12 Askar SM, Saber IM and Omar M: Mastoid reconstruction with platelet-rich plasma and bone pate after canal wall down mastoidectomy: A preliminary report. Ear Nose Throat J, 2019. PMID: 31581830. DOI: 10.1177/0145561319879789

13 Yamamoto Y, Takahashi K, Morita Y, Ohshima S and Takahashi S: Long-term follow-up results of canal wall down tympanoplasty with mastoid obliteration using the bone pate plate for canal wall reconstruction in cholesteatoma surgery. Otol Neurotol 35(6): 961-965, 2014. PMID: 24853244.

14 Moore ST, Katz JM, Zhukauskas RM, Hernandez RM, Lewis CS, Supronowicz PR, Gill E, Grover SM, Long NS and Cobb RR: Osteoconductivity and osteoinductivity of puros ${ }^{\circledR}$ DBM putty. J Biomater Appl 26(2): 151-171, 2011. PMID: 20566657. DOI: $10.1177 / 0885328210366061$

15 Schaefer D, Martin I, Shastri P, Padera RF, Langer R, Freed LE and Vunjak-Novakovic G: In vitro generation of osteochondral composites. Biomaterials 21(24): 2599-2606, 2000. PMID: 11071609. DOI: $10.1016 / \mathrm{s} 0142-9612(00) 00127-7$

16 Chamberlain G, Fox J, Ashton B and Middleton J: Concise review: Mesenchymal stem cells: Their phenotype, differentiation capacity, immunological features, and potential for homing. Stem Cells 25(11): 2739-2749, 2007. PMID: 17656645.

17 Breen A, O'Brien T and Pandit A: Fibrin as a delivery system for therapeutic drugs and biomolecules. Tissue Eng Part B: Rev 15(2): 201-214, 2009. PMID: 19249942.

18 Abiraman S, Varma H, Umashankar P and John A: Fibrin glue as an osteoinductive protein in a mouse model. Biomaterials 23(14): 3023-3031, 2002. PMID: 12069345.

19 Glassman SD, Howard J, Dimar J, Sweet A, Wilson G and Carreon L: Complications with recombinant human bone morphogenic protein-2 in posterolateral spine fusion: A consecutive series of 1037 cases. Spine 36(22): 1849-1854, 2011. PMID: 20838369. DOI: 10.1097/BRS.0b013e3181d133d0
20 Kurzyk A, Ostrowska B, Święszkowski W and Pojda Z: Characterization and optimization of the seeding process of adipose stem cells on the polycaprolactone scaffolds. Stem Cells Int 2019: 1201927, 2019. PMID: 30915123. DOI: 10.1155/2019/1201927

21 Kasten P, Luginbuhl R, van Griensven M, Barkhausen T, Krettek $\mathrm{C}$, Bohner $\mathrm{M}$ and Bosch $\mathrm{U}$ : Comparison of human bone marrow stromal cells seeded on calcium-deficient hydroxyapatite, betatricalcium phosphate and demineralized bone matrix. Biomaterials 24(15): 2593-2603, 2003. PMID: 12726713. DOI: 10.1016/s0142-9612(03)00062-0

22 Kang EJ, Byun JH, Choi YJ, Maeng GH, Lee SL, Kang DH, Lee JS, Rho GJ and Park BW: In vitro and in vivo osteogenesis of porcine skin-derived mesenchymal stem cell-like cells with a demineralized bone and fibrin glue scaffold. Tissue Eng Part A 16(3): 815-827, 2010. PMID: 19778183. DOI: 10.1089/ten. TEA.2009.0439

23 Marden LJ, Hollinger JO, Chaudhari A, Turek T, Schaub RG and Ron E: Recombinant human bone morphogenetic protein-2 is superior to demineralized bone matrix in repairing craniotomy defects in rats. J Biomed Mater Res 28(10): 1127-1138, 1994. PMID: 7829543. DOI: $10.1002 / \mathrm{jbm} .820281003$

24 Groeneveld EH, van den Bergh JP, Holzmann P, ten Bruggenkate $\mathrm{CM}$, Tuinzing DB and Burger EH: Mineralization processes in demineralized bone matrix grafts in human maxillary sinus floor elevations. J Biomed Mater Res 48(4): 393-402, 1999. PMID: 10421679.

25 James AW, LaChaud G, Shen J, Asatrian G, Nguyen V, Zhang $\mathrm{X}$, Ting $\mathrm{K}$ and Soo C: A review of the clinical side-effects of bone morphogenetic protein-2. Tissue Eng Part B Rev 22(4): 284-297, 2016. PMID: 26857241. DOI: 10.1089/ten.TEB. 2015.0357

Received January 27, 2020

Revised March 2, 2020

Accepted March 5, 2020 